\title{
A STUDY OF SMOKING BEHAVIOUR AMONG MALES WITH SCHIZOPHRENIA
}

\author{
Thirumalai Ranganathan ${ }^{1}$ \\ ${ }^{1}$ Senior Assistant Professor, Department of Psychiatry, Madras Medical College.
}

\begin{abstract}
BACKGROUND

Nicotine dependence is the commonest substance use disorder among individuals with schizophrenia. The common co-occurrence of nicotine use and schizophrenia is a manifestation of shared underlying neurobiology. Several studies point towards linkages between the effects of nicotine and the neurobiology of schizophrenia. However, studies like these have all come from the developed west with a different sociocultural milieu. It would be of importance to study smoking by patients with schizophrenia in the non-western developing countries like India. Such studies could evidence whether high smoking in schizophrenia is a universal phenomenon and also help clarify the role of cultural and other non-biological factors on the smoking behaviour. Aim- To study the relationship between smoking status and clinical characteristics in males with schizophrenia.
\end{abstract}

\section{MATERIALS AND METHODS}

75 consenting patients with schizophrenia each of those who smoked and those who did not were recruited for the study at the outpatient department of Institute of Mental Health, Chennai. Patients were assessed using well-validated scales.

\section{RESULTS}

Smokers with schizophrenia had a significantly greater number of hospitalisations, longer duration of hospitalisation, higher PANSS scores, greater depression and anxiety when compared to non-smokers. Smokers also received a significantly higher mean dosage of antipsychotic drugs than non-smokers. Smoking status had a high positive correlation with positive syndrome, negative syndrome, general psychopathology, high akathisia scores and antipsychotic dose.

\section{CONCLUSION}

Smoking status is associated with a severe psychiatric illness. The clinical implications of this study are: a) Smoking status should be addressed when evaluating data from studies of antipsychotic dose, efficacy and side effects; b) Smoking possibly reflects selfmedication of underlying psychopathology or of medication side effects.

\section{KEYWORDS}

Smoking, Schizophrenia, Nicotine Dependence.

HOW TO CITE THIS ARTICLE: Ranganathan T. A study of smoking behaviour among males with schizophrenia. J. Evolution Med. Dent. Sci. 2017;6(45):3534-3541, DOI: 10.14260/Jemds/2017/762

\section{BACKGROUND \\ The use of tobacco to create and satisfy nicotine addiction was introduced to Columbus by Native Americans and spread rapidly to Europe. The use of tobacco as cigarettes, however, is predominantly a twentieth century phenomenon, as is the epidemic of disease caused by this form of tobacco. \\ Several sociocultural and economic factors could influence smoking behaviour among the Indian population. In our country, smoking is often prohibited by familial, cultural and religious practices. For example, less than 3\% of Sikh men living in urban Delhi were found to be smokers compared to $45 \%$ of men in general. The cost factor is another important one that determines the frequency and severity of smoking, especially among the poor.[1]}

Financial or Other, Competing Interest: None.

Submission 25-04-2017, Peer Review 25-05-2017,

Acceptance 31-05-2017, Published 05-06-2017.

Corresponding Author:

Dr. Thirumalai Ranganathan,

Senior Assistant Professor,

Department Psychiatry,

Madras Medical College,

Chennai- 600020.

E-mail: drrangagh@gmail.com

DOI: $10.14260 /$ jemds $/ 2017 / 762$

\section{(c) $(1)(5)$}

Nicotine use is extremely common among individuals with schizophrenia. Beginning in 1983, published reports described the rate of current smoking as dramatically elevated among patients with schizophrenia. Early reports described the prevalence of smoking as up-to 88\%.[2] Hughes et al (1986) examined smoking rates among a relatively large $(n=277)$ young adult, outpatient psychiatric population and compared these rates among local and national population based samples. That study was the first to provide data to support the hypothesis that increased smoking rates are specifically related to psychiatric diagnoses, even when other contributing factors are controlled. The rate of smoking was highest among patients with schizophrenia (88\%), compared with those with mania (70\%), major depressive disorder (49\%), and anxiety, personality, or adjustment disorder diagnoses (45-47\%) and with the control population (30\%).[3]

In the general population, smoking prevalence is greater among men and is inversely related to age, education, and socioeconomic status. Although they have been incompletely examined, among individuals with schizophrenia, the relationships of smoking with gender, age, and socioeconomic status appear to persist. $[4,5,3]$

There are few Indian studies in this area of research. A study in India on 286 urban male outpatients with schizophrenia, only $38 \%$ were found to be current smokers. This was significantly more than in other psychiatric patients 
studied (Major affective disorders and non-psychotic disorders), but not medically ill controls and not higher than the rates for the general male population in India.

These authors suggest that the biology of the illness may not be the only factor that relates to the rate of smoking in schizophrenia, considering the influence culture and social practices could have upon the smoking behaviour. Nonbiological factors like lack of economic independence and restrictions imposed by the family appeared to be crucial factors that controlled the prevalence of smoking among patients with schizophrenia in this study.[6]

\section{Aim}

To study the relationship between smoking status and clinical characteristics in males with schizophrenia.

\section{Objectives}

a. To describe the socio-demographic and other non-clinical characteristics of patients with schizophrenia who smoke and to compare these with those who do not.

b. To compare the psychopathology among smokers and non-smokers with schizophrenia.

c. To assess and to compare the antipsychotic related side effects among smokers and non-smokers with schizophrenia.

\section{MATERIALS AND METHODS}

It is a descriptive comparative study. The study describes the clinical characteristics of male patients with schizophrenia who smoke and compares them with those who do not. Women were excluded from this study because of the very low baseline levels of smoking in them, reported in India. The study protocol was presented and permission to conduct the study was obtained from the research and ethics committee of the Institute of Mental Health, Madras Medical College, Chennai.

This study was conducted at the outpatient department of the Institute of Mental Health, Madras Medical College, Chennai. Seventy five consecutive patients each, of those who smoked and those who did not, satisfying the inclusion and exclusion criteria were recruited for the study. Informed consent was obtained from all the patients and their relatives for conducting the study.

All persons attending the outpatient department of the Institute of Mental Health, who satisfied the ICD-10 criteria for a diagnosis of schizophrenia, were included in the study. Patients who were unwilling or uncooperative, those who had a comorbid diagnosis, patients having an associated medical/neurological problem, patients on atypical antipsychotics, patients undergoing/those who have underwent smoking cessation/reduction treatment were excluded from this study.

\section{Methods}

Every patient was assessed by a clinical interview using a semi-structured interview schedule, Positive and Negative Syndrome Scale (PANSS), Simpson-Angus rating scale (SAS), Abnormal Involuntary Movements scale (AIMS), Barnes Akathisia Rating Scale, Calgary Depression Rating Scale for Schizophrenia, Hamilton Rating Scale for Anxiety (HAM-A) and Fagerstrom test for nicotine dependence. Each patient was thus interviewed and rated by the examiner for about one hour.

PANSS is a well-validated instrument used to assess and to quantify positive and negative schizophrenia. It includes thirty items on three subscales: seven items covering positive symptoms (hallucinations and delusions), seven covering negative symptoms (e.g. blunted affect) and sixteen covering general psychopathology (e.g. guilt, uncooperativeness). Each item is scored on a seven-point, item-specific Likert scale ranging from 1 to 7 ; thus, the positive and negative subscales each range from 7 to 49 , and the general psychopathology scale from 16 to 112 . SAS is used to assess the extrapyramidal side effects of antipsychotic drugs. It has ten items, each of which is rated on an item-specific, five-point severity scale from 0 to 4 . AIMS is a clinical examination and rating scale used to measure dyskinetic symptoms in patients taking antipsychotic drugs. It has 12 items, each of which is rated on an item-specific, five-point severity scale ranging from 0 to 4 . Barnes akathisia rating scale is used to assess akathisia both objectively and subjectively on a 3-point severity scale ranging from 0 to 3 . Calgary depression scale of schizophrenia is a 9-item scale developed specifically for the assessment of depression in schizophrenia. Each item is scored on a scale of 0 to 3; anchor point descriptions being provided to delineate each item score. HAM-A has 14 items, each of which is rated 0 to 4 on an unanchored severity scale, with the total score ranging from 0 to 56. Fagerstrom test for nicotine dependence measures the severity of nicotine dependence. It is a seven-item scale, yielding a maximum total score of 11 . All patients received a consensus diagnosis by two independent consultants at the outpatient department of the Institute of Mental Health. Patients were considered current smokers if they reported smoking a minimum of five cigarettes/bidis over the past one month. Non-smokers were those who had never smoked cigarettes/bidis. Cigarettes and bidis were considered the same for the sake of convenience in this study. Whenever possible, the patient's history was corroborated by the medical record, clinicians, and family members. Patients who had discontinued medications during the past one month were not included in study. Statistical analysis was done using SPSS (statistical package for social science) version 22.0. Descriptive statistics are shown as mean and standard deviation values. Independent sample test and chi-squared tests were applied. Significance level was fixed as $\mathrm{p}<0.05$.

\section{RESULTS}

A total of one hundred and fifty patients with schizophrenia were interviewed for the present study. The sociodemographic description of the sample is summarised in table 1 and depicted in figures 1 and 2 . The non-smokers had a mean age \pm S.D. of $31.79 \pm 9.01$ years and the smokers had a mean age of $34.08 \pm 8.94$ years. Hindus constituted $92 \%$ and $88 \%$, respectively of smokers and non-smokers. $5.3 \%$ of nonsmokers where Christians compared to only $1.3 \%$ of the smokers. $6.7 \%$ of patients belonged to the Muslim religion in both the groups. $52 \%$ of non-smokers and $57.3 \%$ of the smokers hailed from the rural areas. Smokers had an average education of $9.81 \pm 4.36$ years, when compared to the nonsmokers who had only $7.95 \pm 3.81$ years. Majority of the sample belonged to the low socioeconomic status. The employment status revealed that $8 \%$ of smokers were in a 
regular employment, $4 \%$ were daily labourers, $5.3 \%$ were self-employed and $81.3 \%$ of them were unemployed. In comparison among the non-smokers, $10.7 \%$ were in a regular employment, $9.3 \%$ were daily labourers, $13.3 \%$ were selfemployed and $62.7 \%$ were unemployed. $30.7 \%$ of the smokers were married when compared to $25.3 \%$ of the nonsmokers. $64.1 \%$ of the non-smokers and $50.7 \%$ of the smokers, had never married. $9.3 \%$ of non-smokers and $18.6 \%$ of the smokers had divorced. $68 \%$ of the smokers and $65.3 \%$ of non-smokers lived in a joint family system. While $2.7 \%$ of the smokers lived alone, only $1.3 \%$ of non-smokers were living alone on the platform.

\section{Details of Smoking Behaviour (Table 2)}

$25.3 \%$ of patients smoked between 21 and 30 cigarettes and $24 \%$ smoked $>51$ cigarettes per day. The age at onset of smoking ranged from 9 years to 39 years with a mean + S.D. of $17.99 \pm 6.52$ years. Patients had smoked cigarettes for an average of $16.14 \pm 10.64$ years. Scores on the Fagerstrom Test for Nicotine dependence ranged from 6 to 11 with an average of $9.56 \pm 1.45$. While $36 \%$ of the smokers scored 11 , only $9.3 \%$ had scored seven on the Fagerstrom test. $74.7 \%$ of the smokers had started using tobacco before onset of the psychotic Illness. $53.3 \%$ of the patients were heavy smokers (> 31 cigarettes per day).

\section{Clinical Data}

Table 3 shows the summary of the various clinical data of the two groups. The mean age of onset of psychotic illness for the non-smokers was $24.15 \pm 5.95$ years, when compared to the smokers who had the onset at $24.16 \pm 5.89$ years. While the mean age at first psychiatric consultation/hospitalisation was $26.81 \pm 6.6$ years for the non-smokers, it was $27.84 \pm 7.31$ years for the smokers. The average of total duration of psychotic illness was $7.64 \pm 5.83$ years for the non-smokers, while the smokers had an average duration of $9.87 \pm 9.17$ years of illness. The non-smokers were hospitalised on an average $1.01 \pm 1.56$ times and were in the hospital for $24.12 \pm$ 48.30 days. However, the smokers had been hospitalised for a mean duration of $47.72 \pm 75.13$ days and an average of $2.47 \pm$ 4.00 number of times to the institution, at the time of the interview. While $33.3 \%$ of the smokers had a family history of mental illness, only $20 \%$ of non-smokers had such a history. $69.3 \%$ of the smokers had a family history of substance use, compared to only $12 \%$ of the non-smokers who gave a similar history in their family. $42.7 \%$ of the smokers used tobacco in some other form, in addition to smoking it. However, only $8 \%$ of the non-smokers used tobacco in other modes $(83.3 \%$ of them were using snuff and the rest chewed tobacco). The smokers drank an average of $9.67 \pm 7.46$ cups of coffee per day, when compared to $2.03 \pm 1.52$ cups consumed by the non-smokers. A clinical assessment of insight revealed that while $90.7 \%$ of the smokers denied their illness, only $48 \%$ of the non-smokers totally lacked insight. Patients who smoked received a mean \pm SD of $646.33 \pm$ $150.54 \mathrm{mg}$ of antipsychotics (Converted into Chlorpromazine equivalents) in the past one month. Non-smokers, however, had received a mean of $390.00+170.06 \mathrm{mg}$ of Chlorpromazine equivalents.

\section{Comparison between the Two Groups}

There were no statistically significant differences between smokers and non-smokers on the age, income, religion, education, employment status and place of residence. However, it was observed that the non-smokers were slightly younger, were better educated, had an urban background and had a regular employment, when compared with the smokers. A greater number of smokers (30.7\%) were married when compared with non-smokers (25.3\%). This difference in the marital status was, however, not statistically significant.

Smokers had a heavier genetic loading, in the form of greater family history of mental illness, when compared to the non-smokers. This difference was, however, not significant. Table 4 shows the summary of the comparisons between the groups. There was a significantly higher family history of substance abuse among the smokers when compared with the non-smokers $(P=0.001)$. The two groups did not have any statistically significant differences in the age at onset of psychotic illness, age at first hospitalisation /psychiatric consultation, and the duration of illness. Smokers had a significantly greater number of hospitalisations $(\mathrm{P}=0.004)$. They were also hospitalised for a significantly longer duration than the non-smokers $(P=0.024)$. Significantly higher number of smokers were found to be using tobacco in other forms (In addition to smoking cigarettes), when compared to the non-smokers $(\mathrm{P}=0.001)$. Current smokers also reported drinking more caffeine per day $(\mathrm{P}=0.001)$. Current smokers obtained statistically significant higher scores on the PANSS than nonsmokers. Smokers were found to have a higher positive syndrome, negative syndrome, general psychopathology and total psychopathology scores. Analysis of the five factor scores on the PANSS revealed that the smokers had significantly higher anergia, thought disturbance, activation, paranoid-belligerence, and depression scores. It was found that the smokers had significantly higher scores on the Hamilton rating scale for anxiety. However, both the groups scored below 14, which has been suggested as the threshold for clinically significant anxiety.

Smokers had received a higher mean dosage of antipsychotic drugs (in terms of chlorpromazine equivalents) and this difference was found to be statistically significant $(\mathrm{P}=0.001)$. The two groups did not differ in mean weight or in the frequency of concurrently prescribed anti-cholinergic medication. Current smokers displayed significantly less Parkinsonism (Lower Simpson-Angus Scale scores) but higher levels of akathisia (higher Barnes Akathisia Rating Scale Scores). Smokers were also found to have scored higher than the non-smokers on the Abnormal Involuntary Movement Scale. This difference was not, however, found to be statistically significant.

$53.3 \%$ of the sample $(n=40)$ were heavy smokers. A comparison between "heavy" and "light" smokers revealed that the two groups did not significantly differ in sociodemographic variables. Statistically significant differences were not observed between the groups on different illness variables like, age at onset of psychoses, duration of illness, number of hospitalisations and on the duration of hospitalisation. Heavy smokers were found to have a significantly higher positive syndrome score on PANSS $(\mathrm{P}=0.001)$. 
They also consumed greater amounts of caffeine than the light smokers $(P=0.001)$. Analysis of the five factors in the PANSS revealed that the heavy smokers had significantly higher scores on thought disturbance $(\mathrm{P}=0.003)$, activation $(\mathrm{P}=0.04)$, and paranoid-belligerence $(\mathrm{P}=0.025)$. The heavy smokers were also found to have significantly higher scores on the Calgary depression scale $(\mathrm{P}=0.034)$. Although the heavy smokers had a higher score on the Abnormal Involuntary Movements Scale than the light smokers, the difference was not statistically significant.

Patients who had started smoking before the onset of the psychoses were compared with those who had started smoking after the onset of the illness. It was found that the two did not differ significantly in any of the variables except anergia. Patients who started smoking after the onset of the illness were found to have significantly higher anergia scores $(\mathrm{P}=0.023)$.

Analysis using the Pearson's correlation statistics, revealed that the smoking status had a high positive correlation with positive syndrome, negative syndrome, general psychopathology, and the PANSS factors, anergia and activation. Smoking status also correlated highly with Barnes akathisia scores, antipsychotic dose and coffee use. Caffeine intake was significantly correlated with smoking status( $\mathrm{r}=$ 0.581), Positive syndrome $(\mathrm{r}=0.571)$, General psychopathology $(r=0.525)$ and activation $(r=0.562)$ scores on the PANSS. It was not significantly correlated with the negative syndrome subscale of PANSS and Barnes akathisia score. Antipsychotic dose was significantly correlated to positive syndrome $(r=0.630)$, negative syndrome $(r=0.561)$, activation $(r=0.607)$, and akathisia scores $(r=0.502)$.

\begin{tabular}{|c|c|c|}
\hline Label & $\begin{array}{c}\text { Non-Smokers } \\
\text { (n=75) }\end{array}$ & $\begin{array}{c}\text { Smokers } \\
\text { (n=75) }\end{array}$ \\
\hline Family Income & 1,179 & 1,461 \\
Mean Rs. & & \\
\hline Religion (\%) & 88 & 92 \\
Hindu & 6.7 & 6.7 \\
Muslim & 5.3 & 1.3 \\
Christian & 10.7 & 8 \\
\hline Occupation (\%) & 9.3 & 4 \\
Regular & 13.3 & 5.3 \\
Daily Labourer & 4 & 1.3 \\
Self-Employed & 62.7 & 81.3 \\
Student & & \\
Unemployed & 64.1 & 50.7 \\
\hline Marital Status (\%) & 25.3 & 30.7 \\
Never Married & 9.3 & 18.6 \\
Married & 1.3 & 0 \\
Divorced & & \\
Widowed & 48 & 42.7 \\
\hline Residence (\%) & 52 & 57.3 \\
Urban & 65.3 & 28.0 \\
Rural & 32.0 & 1.3 \\
\hline Family System (\%) & 1.4 & \\
Joint & & \\
Nuclear & & \\
Extended & Table 1. Socio-demographic Data \\
Alone & & \\
\hline \multicolumn{2}{|c|}{} & \\
\hline
\end{tabular}

\begin{tabular}{|c|c|}
\hline $\begin{array}{c}\text { Number of Cigarettes } \\
\text { Smoked Per Day (\%) } \\
10-20\end{array}$ & $16(21.3 \%)$ \\
$21-30$ & $19(25.3 \%)$ \\
$31-40$ & $13(17.3 \%)$ \\
$41-50$ & $9(12 \%)$ \\
$\geq 51$ & $18(24 \%)$ \\
\hline Age at onset of smoking, & $17.99(6.52)$ \\
mean (SD) & $16.14(10.64)$ \\
\hline Number of years of smoking, & \\
mean (SD) & $56(74.7)$ \\
\hline Onset of smoking, n (\%) & $19(25.3)$ \\
Before the illness & \\
After the illness & $2(2.6)$ \\
\hline Fagerstrom test score, n & $7(9.3)$ \\
(\%) & $9(12)$ \\
7 & $13(17.3)$ \\
8 & $17(22.7)$ \\
9 & $27(36)$ \\
10 & \\
11 & \\
\hline Table 2. Details of Smoking Behaviour \\
\hline
\end{tabular}

\begin{tabular}{|c|c|c|c|c|}
\hline \multirow{2}{*}{ Label } & \multicolumn{4}{|c|}{ Smoking Status } \\
\cline { 2 - 5 } & Non-Smokers & \multicolumn{2}{c|}{ Smokers } \\
\cline { 2 - 5 } & Mean & SD & Mean & SD \\
\hline Age at onset of illness & 24.15 & 5.95 & 24.16 & 5.89 \\
\hline $\begin{array}{c}\text { Age at first } \\
\text { hospitalisation }\end{array}$ & 26.81 & 6.60 & 27.84 & 7.31 \\
\hline Duration of illness & 7.64 & 5.83 & 9.87 & 9.17 \\
\hline $\begin{array}{c}\text { Number of } \\
\text { hospitalisation }\end{array}$ & 1.01 & 1.56 & 2.47 & 4.00 \\
\hline $\begin{array}{c}\text { Duration of last } \\
\text { hospitalisation }\end{array}$ & 24.12 & 48.30 & 47.71 & 75.13 \\
\hline $\begin{array}{c}\text { Number of cups of } \\
\text { coffee }\end{array}$ & 2.03 & 1.52 & 9.67 & 7.46 \\
\hline $\begin{array}{c}\text { Chlorpromazine } \\
\text { equivalents (mg) }\end{array}$ & 390.00 & 170.06 & 646.33 & 150.54 \\
\hline \multicolumn{4}{|c|}{ Table 3. Clinical Data } \\
\hline
\end{tabular}

\begin{tabular}{|c|c|c|c|c|c|c|c|}
\hline \multirow{3}{*}{ Item } & \multicolumn{4}{|c|}{ Smoking Status } & \multirow{3}{*}{ 't' } & \multirow{3}{*}{ df } & \multirow{3}{*}{ 'p' } \\
\hline & \multicolumn{2}{|c|}{$\begin{array}{c}\text { Non- } \\
\text { Smokers }\end{array}$} & \multicolumn{2}{|c|}{ Smokers } & & & \\
\hline & Mean & SD & Mean & SD & & & \\
\hline $\begin{array}{l}\text { Number of } \\
\text { hospitali- } \\
\text { sations }\end{array}$ & 1.01 & 1.56 & 2.47 & 4.0 & 2.930 & 148 & $0.004^{*}$ \\
\hline $\begin{array}{c}\text { Duration of } \\
\text { hospitali- } \\
\text { sations }\end{array}$ & 24.12 & 48.3 & 47.72 & 75.13 & 2.288 & 148 & $0.024^{*}$ \\
\hline Coffee use & 2.03 & 1.52 & 9.67 & 7.46 & 8.687 & 148 & $0.001^{*}$ \\
\hline $\begin{array}{c}\text { Family history } \\
\text { of substance } \\
\text { use }\end{array}$ & 9 & 66 & 52 & 23 & 51.087 & 1 & $0.001^{*}$ \\
\hline $\begin{array}{l}\text { Other modes } \\
\text { of tobacco use }\end{array}$ & 6 & 69 & 32 & 43 & 23.825 & 1 & $0.001^{*}$ \\
\hline
\end{tabular}

Table 4. Comparison on Illness Variables

* 'p' value significant 

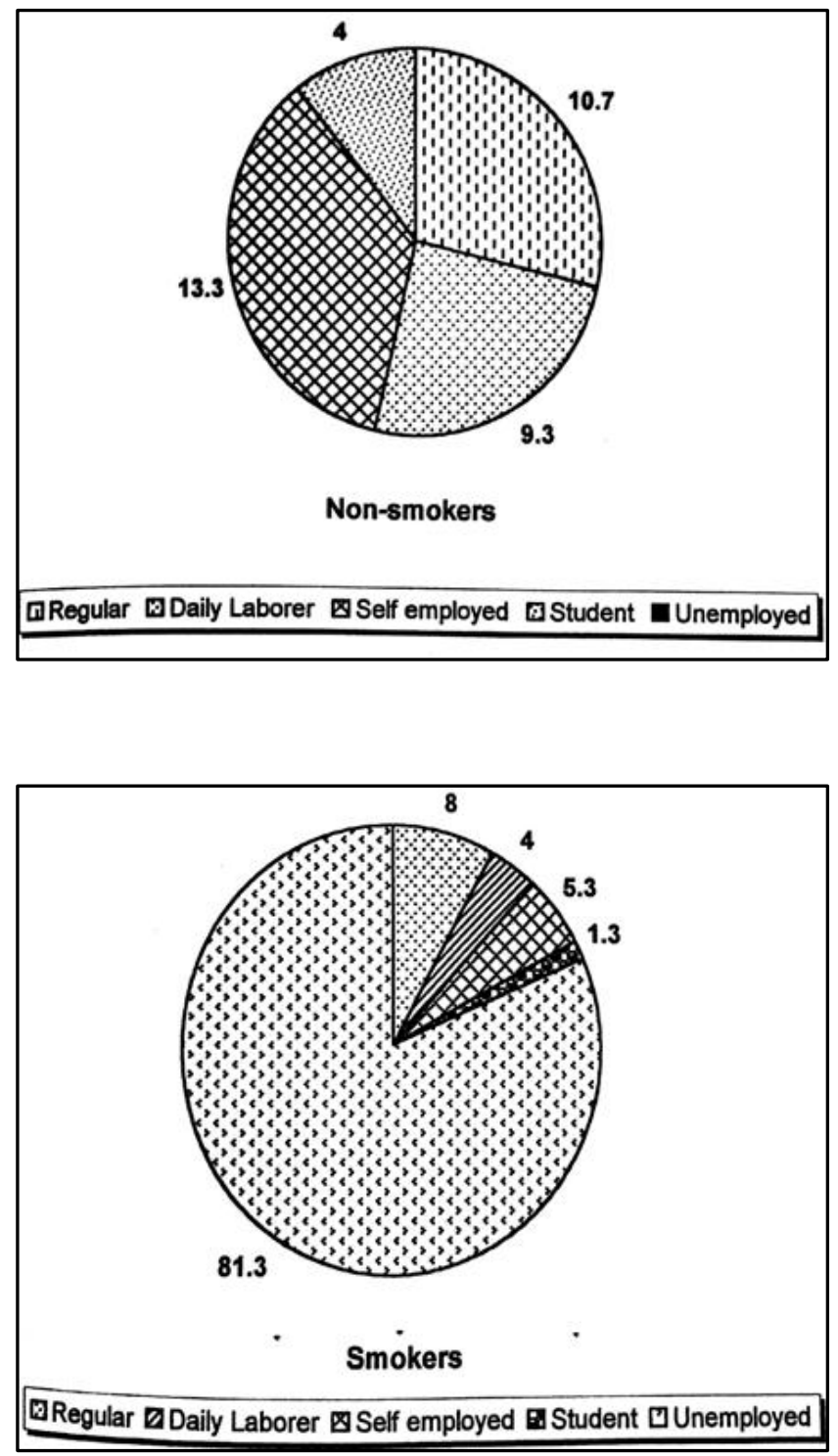

Figure 1. Occupation

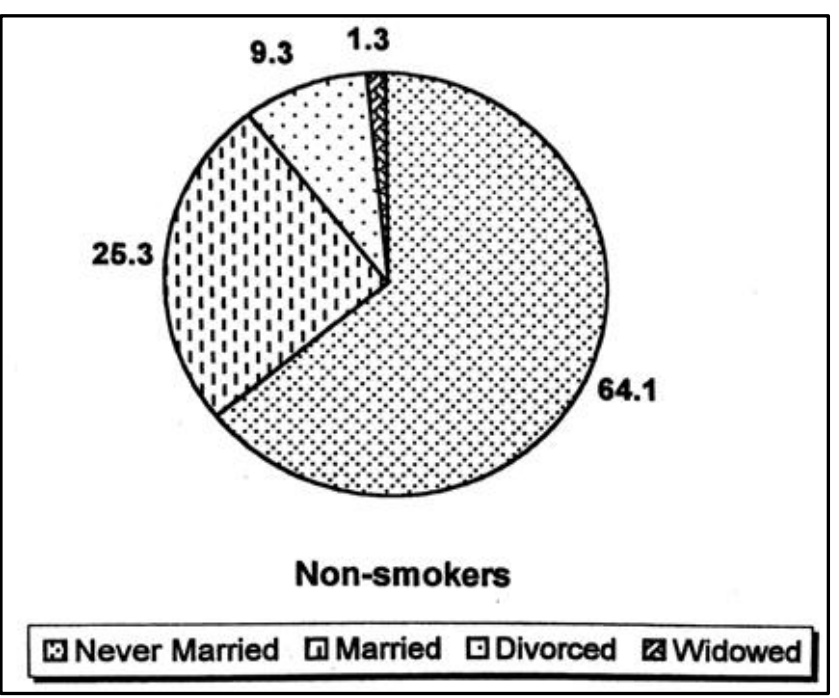

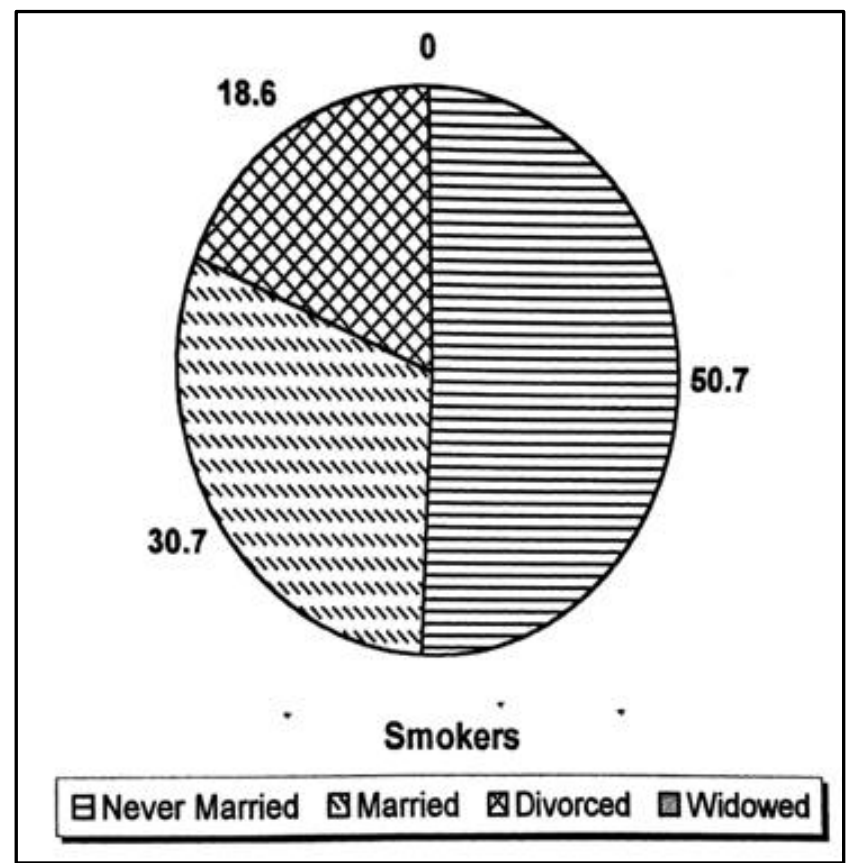

Figure 2. Marital Status

\section{DISCUSSION}

Several western studies have consistently reported high rates of smoking among patients with schizophrenia. These patients belong to different sociocultural and economic backgrounds. Studies from the west often lead us to presume that heavy smoking is an inevitable feature of schizophrenia. The Indian study by Srinivasan and Thara (2002) has addressed this issue and has concluded that smoking in schizophrenia was not more than in the general population. These authors have suggested that non-biological factors are likely to play an important role in modulating smoking behaviour among the Indian patient population. There are, however, no studies in the Indian context which compare the clinical and non-clinical characteristics of patients who smoke with those who do not. This study is an attempt in that direction.

Rabinowitz et al (1998) had reported that the substance use disorder predated the onset of psychosis, in almost all the psychotic patients, in their sample.[7] It was observed that $74.7 \%$ of smokers in this sample had started using tobacco before the onset of their psychotic illness. A comparison of the patients who had started smoking before the onset of the illness with those who had started after the illness, revealed that the former had a younger but statistically nonsignificant, mean age at onset of illness. Moreover, these patients had a significantly higher score on anergia factor of the PANSS, slightly higher negative syndrome subscale scores (which was however not significant), when compared to the non-smokers. The above findings are consistent with the known fact that early age at onset of schizophrenia is associated with more prominent negative symptoms, which in turn is due to decreased dopaminergic function in the limbic system. 
Smokers were also observed to consume significantly greater amounts of caffeine than the non-smokers. The heavy smokers had a significantly higher intake of caffeine than the light smokers and the non-smokers. There was a high correlation between cigarette smoking and caffeine intake. Caffeine intake was also significantly correlated with the positive syndrome subscale and activation scores of PANSS. This is consistent with the reports of previous studies. Lucas et al (1990) had reported that acute administration of caffeine to patients with schizophrenia elevated the scores on the Brief Psychiatric Rating Scale, including positive symptoms such as thought disorder and unusual thought content. The high correlation between caffeine intake and activation can be interpreted as a pointer towards greater hostility and irritability in these patients. ${ }^{[8]}$ Koczapski et al (1989), had found significant reductions in hostility and suspiciousness when de-caffeinated coffee was introduced for in-patients. These changes, however, were reversed when regular coffee was re-introduced.[9] Although caffeine use was associated with elevations of psychosis in this study sample, there is little evidence to suggest that caffeine directly contributed to a worsening of clinical status of our patients.

Smokers in this study, were admitted to the hospital for a greater duration of time and had been committed to hospital more number of times than the non-smokers. These findings were already reported by Goff et al $1992^{[5]}$ and Ziedonis et al, 1994.[10] Smokers in this sample were prescribed antipsychotics at roughly twice the daily dose prescribed for the non-smokers. This finding is probably due to a smoking induced elevation of hepatic clearance of antipsychotic drugs described in previous studies by Jann et al 1986[11] Ereshefsky et al 1985.[12] Patients who smoked also had higher mean scores on the akathisia rating scale, when compared to the non-smokers. Thus, it may be inferred that antipsychotic activity levels in smokers were higher than levels in non-smokers. This inference is, however, limited by the fact that plasma concentrations of the drugs in the two patient groups were not estimated in this study.

The dose of antipsychotics was significantly correlated with the positive syndrome, negative syndrome and activation scores on the PANSS. Patients who smoked, scored significantly high scores on all the PANSS subscales of positive syndrome, negative syndrome, general psychopathology and total psychopathology. A high correlation was also observed between smoking status and the positive syndrome, negative syndrome, general psychopathology and activation scores of PANSS. These findings are consistent with those of Goff et al 1992[5] and Ziedonis et al 1994.[10] Heavy smokers had significantly high scores on the positive syndrome, thought disturbance, activation, paranoid-belligerence subscales of the PANSS than the light smokers and the non-smokers. This study replicates the report of Ziedonis et al 1994[10] with the exception of the finding that the heavy smokers had lower number of negative symptoms than the light smokers. The higher degree of psychiatric impairment found in the smokers of this study may also reflect levels of impulse control insufficient to stop smoking as well as a lack of motivation. The higher doses of antipsychotics received by the smokers could reflect the clinician's efforts to control resistant psychotic symptoms.
Thus, smoking status in schizophrenia could be associated with a vicious cycle of higher dosage of drugs, increased amount of smoking and a higher psychopathology.

Smokers displayed significantly less antipsychoticinduced Parkinsonism as measured by the Simpson-Angus Scale. This finding is consistent with those of the studies by Decina et al, 1990[13] and Sandyk, 1993.[14] This lesser frequency of parkinsonism was observed in the sample despite smokers receiving a two-fold higher dose of antipsychotics and in the presence of higher levels of akathisia.

Previous studies by Wagner et al 1988 had found lower levels of parkinsonism only in smokers with a duration of illness less than seven years.[15] This study has not replicated the above findings. In this sample, the mean duration of illness of smokers was about ten years, and the duration of illness did not correlate with the ratings of Parkinsonism. It is not certain whether cigarette smoking directly affects Parkinsonism. Smoking in the general population appears to protect against the onset of idiopathic Parkinsonism at a later age. Majority of the smokers (75\%) in this sample had begun smoking on an average 8.1 years before being started on antipsychotic therapy. This temporal relationship argues in favour of a possible anti-Parkinsonism effect of smoking, rather than a tendency for patients to smoke in response to the extrapyramidal symptoms. Moreover, smoking induced reduction in antipsychotic plasma levels may not account entirely for this effect. In addition, there were no differences in the anti-cholinergic treatment received by the smokers and non-smokers. Thus, anti-Parkinsonian drugs alone may not explain the low occurrence of Parkinsonism observed among the smokers in this study.

In contrast to previous studies by Yassa et al 1987,[16] Goff et al 1992,[5] this study did not find any significant difference in the Abnormal Involuntary Scale scores between the two groups, though it was observed that the smokers had a marginally higher score. This finding was consistent with those reported by Menza et al 1991[17] who had found no difference between the two groups. The heavy smokers in this sample were found to have obtained a higher score than the light smokers and the non-smokers on the Abnormal Involuntary Movement Scale. This finding, though not statistically significant, is consistent with the reports of Nilsson et al 1997, who had found the risk of dyskinesias to increase with the number of cigarettes smoked per day.[18]

Studies in the past have demonstrated that the rates of nicotine dependence were four times higher than the general population in subjects with major depression and anxiety disorders.[19] This study had found significantly higher rates of depression and anxiety among patients who smoked, compared to those who did not. The heavy smokers were found to have significantly higher scores on the Calgary depression scale than the light smokers and the non-smokers. These findings are at variance with those reported by Goff et al 1992.[5] They had found that the smokers in their sample did not score higher than non-smokers on depression or tension items of the Brief Psychiatric Rating Scale, despite scoring highly on the positive and negative symptoms. Persons with a history of affective illness are more likely to smoke because of the anti-depressant effect of nicotine and 
are more unlikely to stop smoking because abstinence may trigger depressive symptoms. It is also believed that nicotine has anxiolytic properties. Thus, the higher association of smoking and depression may point towards an attempt by these patients to self-medicate their symptoms. It is also possible that smoking selectively improves affective symptoms in these patients. This finding is clinically important, especially, when these patients are taken up for smoking cessation programs. These patients may have difficulties in giving up smoking, as any attempt to quit may soon be followed by worsening of the affective and anxiety symptoms.

\section{CONCLUSION}

Smoking status is associated with a severe psychiatric illness. This is evidenced by the higher psychopathology and poorer social and occupational functioning of these patients, when compared with the non-smokers. Smoking may also be considered as a marker of increased psychiatric symptoms in patients with schizophrenia. Smokers have lesser extrapyramidal side effects, in spite of receiving higher dosages of antipsychotics. Smokers may also have a tendency to develop more abnormal involuntary movements than the non-smokers. Heavier the smoking, more is the likelihood of developing dyskinesias. Smoking may increase synaptic levels of dopamine leading to a higher psychopathology and an increase in antipsychotic dosage. This increase in synaptic dopamine could also be reflected by the lower levels of Parkinsonism among the smokers.

\section{The Clinical Implications of this Study are-}

a. Smoking status should be addressed when evaluating data from studies of antipsychotic dose, efficacy and side effects;

b. If smoking in schizophrenia reflects in part selfmedication of underlying psychopathology or of medication side effects, it is important to be cautious in abruptly subjecting these patients to smoke free units, where symptoms of nicotine abstinence may confuse the clinical picture;

c. Smoking is a common problem raised by most care givers and it is very essential for clinicians to understand its implications for the management of these patients.

This study has some important limitations. It is a descriptive comparative study. Hence, it is not possible to draw etiological conclusions. It may also be difficult to generalise the findings of this study. The rater was not blind to the smoking status of the patients. Thus, it is possible that observer bias may have influenced the results. Future studies may a)include drug naïve patients in order to remove the confounding effect of drugs; b) evaluate patients prospectively in order to determine the temporal relationship between smoking behaviour and psychiatric symptoms; c) define the temporal relationship between the last cigarette smoked and evaluation for tardive dyskinesia in order that short-term changes in abnormal involuntary movements may be interpreted with greater clarity; d) identify factors that reinforce smoking behaviour in patients with schizophrenia, so that attempts to reduce their exposure to this serious health hazard may be more successful.

\section{REFERENCES}

[1] Venkatnarayan KM, Chadda SL, Hanson RL, et al. Prevalence and pattern of smoking in Delhi: crosssectional study. British Journal of Medicine 1996;312(7046):1576-9.

[2] O'Farell TJ, Connors GJ, Upper D. Addictive behaviors among hospitalized psychiatric patients. Addictive Behaviors 1983;8(4):329-33.

[3] Hughes JR, Hatsukami DK, Mitchell JE, et al. Prevalence of smoking among psychiatric outpatients. American Journal of Psychiatry 1986;143(8):993-7.

[4] de Leon J, Dadvand M, Canuso C, et al. Schizophrenia and smoking: an epidemiological survey in a state hospital. American Journal of Psychiatry 1995;152(3): 453-5.

[5] Gofff DC, Henderson DC, Amico E. Cigarette smoking in Schizophrenia: relationship to psychopathology and medication side Effects. American Journal of Psychiatry 1992;149(9):1189-94.

[6] Srinivasan TN, Thara R. Smoking in schizophrenia - all is not biological. Schizophrenia Research 2002;56(12):67-74.

[7] Rabinowitz J, Bromet EJ, Lavelle J, et al. Prevalence and severity of substance use disorders and onset of psychosis in first admission psychotic patients. Psychological Medicine 1998;28(6):1411-9.

[8] Lucas PB, Pickar D, Kelsoe J, et al. Effects of the acute administration of caffeine in patients with schizophrenia. Biological Psychiatry 1990;28(1):3540 .

[9] Koczapski A, Paredes J, Kogan C, et al. Effects of caffeine on behavior of schizophrenic in-patients. Schizophrenia Bulletin 1989;15(2):339-44.

[10] Ziedonis DM, Kosten TR, Glaser WM, et al. Nicotine dependence and schizophrenia. Hospital and Community Psychiatry 1994;45(3):204-6.

[11] Jann MW, Saklad SR, Ereshefsky L, et al. Effects of smoking on haloperidol and reduced haloperidol plasma concentrations and haloperidol clearance. Psychopharmacology 1986;90(4):468-70.

[12] Ereshefsky L, Jann MW, Saklad SR, et al. Psychiatric inpatients. Biological Psychiatry 1985;20:329-32.

[13] Decina P, Caracci G, Sandik R, et al. Cigarette smoking and neuroleptic-induced Parkinsonism. Biological Psychiatry 1990;28(6):502-8.

[14] Sandyk R. Cigarete smoking: effects on cognitive functions and drug-induced Parkinsonism in chronic schizophrenia. International Journal of Neuroscience 1993;70(3-4):193-7.

[15] Wagner B, Wolf GK, Ulmar G. Does smoking reduce the risk of neuroleptic parkinsonoids? Pharmacopsychiatry 1988;21(6):302-3.

[16] Yassa R, Lal S, Korpassy A, et al. Nicotine exposure and Tardive dyskinesia. Biological Psychiatry 1987;22(1): 67-72. 


\section{Jemds.com}

[17] Menza MA, Grossman N, VanHorn M, et al. Smoking and movement disorders in psychiatric patients. Biological Psychiatry 1991;30(2):109-15.

[18] Nilsson A, Waller L, Rosengren A, et al. Cigarette smoking is associated with abnormal involuntary movements in the general male population - a study of men born in 1933. Biological Psychiatry 1997;41(6):717-23.
Original Research Article

[19] Breslau N, Kilbey M, Audreski P. Nicotine dependence, major depression and anxiety in young adults. Archives of General Psychiatry 1991;48(12):1069-74. 